\title{
The beneficial effects of individualizing adequate atrioventricular delay in dual chamber pacemakers
}

Volume 5 Issue 3 - 2016

\section{Editorial}

Maintenance of adequate atrioventricular (AV) synchrony is important to preserve cardiac function and to achieve a better prognosis in patients with dual chamber pacemakers (PM). ${ }^{1,2}$ Left ventricular (LV) dysfunction may occur as a result of inappropriate AV delay in some PM patients. Therefore, AV delay optimization is important to preserve LV systolic function and a favorable long-term outcome after sequential pacing. ${ }^{3,4}$ In accordance with the Frank-Starling law, AV delay should be optimized to achieve maximal LV filling without deterioration of LV function. ${ }^{2}$ The purpose of AV delay optimization is to ensure that LV contraction does not occur before complete filling allowing an adequate systolic stroke volume. AV delay optimization is achieved using the Ritter's method which aims at maximizing the LV filling during diastole by allowing for mitral valve closure to occur after a complete atrial systole. The interval between QRS onset and closure of mitral valve is measured by programming short and long AV intervals. Typically, the Doppler A wave is truncated with short AV delay intervals, but long AV delay intervals can cause fusion of the $\mathrm{E}$ and $\mathrm{A}$ waves and mitral valve diastolic regurgitation. Therefore, the optimal AV delay is calculated as the long AV delay minus the difference of the time intervals of the QRS onset to mitral valve closure at short and long AV intervals. ${ }^{5}$ The Optimal AV synchronization will not only maximize cardiac output by increasing ventricular preload and by lowering the mean atrial pressure, but it will also minimize the diastolic mitral regurgitation. The electrocardiogram, as well as, the Doppler echocardiography has been utilized as interesting methods to optimize the AV delay in PM patients. ${ }^{6-14}$ Although feasible and easy to perform, the systematic utilization of these latter techniques still remains controversial.

In this issue of this journal, Aboulenein $\mathrm{J}$ et al., ${ }^{15}$ presented an interesting investigation which aimed to optimize the AV delay in patients with an implanted PM. ${ }^{15}$ They tried to compare the systolic and diastolic performance between the optimized AV delay and the default AV delay of the PM manufacturer, as well as to assess the optimal AV delay for each patient and to decide whether this optimization is worth doing it for all patients. ${ }^{15}$ The authors found a statistically significant increase in the LV ejection fraction (EF) in PM patients with the AV delay optimized by trans-mitral Doppler waves guidance on echocardiography after 12 months of device implantation. Similar results were observed as regard to the right ventricular EF which presented a significant increase. They concluded that optimizing the AV delay for all patients with dual chamber pacemakers may lead to long term beneficial effects mainly on the systolic functions. The authors should be congratulated because unlike other studies, they investigated also the right ventricular systolic function, and found a significant improvement in the AV delay optimized PM patients.

Several interesting other studies shed some light on the proper way of optimizing AV delay in PM patients. Sawhney NS et al., ${ }^{16}$ compared an echo-guided AV delay optimization with an empiric,

\author{
Osmar Antonio Centurión 1,2 \\ 'Department of Health Sciences's Investigation, Sanatorio \\ Metropolitano, Fernando de la Mora, Paraguay \\ ${ }^{2}$ Cardiology Division, First Department of Internal Medicine, \\ Asunci
}

Correspondence: Osmar Antonio Centurión, Professor of Medicine, Asuncion National University, Department of Health Sciences's Investigation, Sanatorio Metropolitano, Cardiology Division, First Department of Internal Medicine, Asunción National University, Teniente Ettiene 215 c/Ruta Mariscal Estigarribia, Fernando de la Mora, Asunción, Paraguay, Email osmarcenturion@hotmail.com

Received: February 24, 2016 | Published: March 04, 2016

fixed AV delay of $120 \mathrm{~ms}$ in a randomized prospective, control trial. They demonstrated improved clinical outcome at 3 months in patients with echo-guided AV optimization. ${ }^{16}$ In their study, optimal AV delay was defined as the largest aortic velocity-time integral at one of eight tested AV intervals (between 60 and $200 \mathrm{~ms}$ ). ${ }^{16}$ A recent large-scale randomized prospective multicenter trial (SMART-AV trial) compared a fixed empirical AV delay (120 ms), an echocardiographically optimized AV delay, and an AV delay optimized with Smart Delay electrocardiogram-based algorithm. ${ }^{17}$ This trial did not show superiority of echocardiography or Smart Delay over a fixed AV delay of $120 \mathrm{~ms} .{ }^{17}$ Ritter et al., ${ }^{18}$ first reported an echo-Doppler method to optimize AV delay in patients with complete AV block and a normal LV systolic function. They defined the AV delay with the echo method that provided the longest diastolic filling time without interruption of the A wave. Ritter's formula, which can be regarded as the current "gold standard" in AV delay optimization ${ }^{19}$ requires 2 measurements. First, the QA short is the time interval between the onset of the Q wave and the end of the truncated " $A$ " wave of the transmitral flow at a short (30-60 ms) AV delay. Second, the QA long is the time interval between the onset of the $\mathrm{Q}$ wave and the end of the " $\mathrm{A}$ " wave of the transmitral flow at a long $(200 \mathrm{~ms}) \mathrm{AV}$ interval. According to the formula, optimal AV delay was calculated as AV long - (QA short - QA long). This method has been used in several clinical trials because it is a simple, non-invasive and a reproducible method. ${ }^{20}$ Due to the timeconsuming process of manual optimization and the lack of guideline recommendations. ${ }^{21,22}$ individualized optimization of the AV delay is not usually performed in the clinical routine practice. Nevertheless, other investigators used different echo Doppler techniques to optimize AV delay. Rossvoll and Hatle ${ }^{23}$ first reported the concept of Doppler assessment of LV filling pressure using both transmitral flow and pulmonary vein flow in $1993 .{ }^{23}$ The difference in duration between the pulmonary vein flow and the antegrade A wave by the transmitral flow was positively and strongly correlated with LV end-diastolic pressure. A longer duration of the pulmonary vein flow versus the A wave predicted an increased LV end-diastolic pressure. ${ }^{23}$ Therefore, an AV delay that does not prolong pulmonary vein flow more than 
the A wave could be considered as a hemodynamically optimal AV delay. In this regard, Fukura $\mathrm{K}$ et al. ${ }^{24}$ demonstrated that AV delay optimization based on an echo-Doppler method using the transmitral flow and pulmonary vein flow is feasible, and significantly increased systolic stroke volume. ${ }^{24}$ They showed that AV delay optimized by this method may suggest a potential favorable impact on cardiac function and possibly prognosis. However, there is a possible limitation with this echo Doppler technique. This method for AV delay optimization was not possible to be performed in some patients in whom pulmonary vein flow could not be detected. Although the sensitivity of the Doppler measurements for some specific conditions was not sufficient when using older echo-Doppler machines, ${ }^{25,26}$ modern echo-Doppler machines have sufficiently sensitive Doppler equipment. ${ }^{27,28}$

The purpose of AV delay optimization is to ensure that LV contraction does not occur before complete filling allowing an adequate systolic stroke volume. AV delay optimization aims at maximizing the LV filling during diastole by allowing for mitral valve closure to occur after a complete atrial systole. AV delay optimization has chronic beneficial hemodynamic effects increasing cardiac output and diminishing mitral regurgitation. Therefore, the role and efficacy of AV delay optimization in improving clinical outcomes seems promising. However, it remains to be investigated whether AV delay optimization may improve long-term survival in larger scale randomized clinical trials.

\section{Acknowledgments}

None.

\section{Conflicts of interest}

Author declares that there is no conflict of interest.

\section{References}

1. Frielingsdorf J, Gerber AE, Hess OM. Importance of maintained atrio-ventricular synchrony in patients with pacemakers. Eur Heart $J$. 1994;15(10):1431-1440.

2. Guardigli G, Ansani L, Percoco GF, et al. AV delay optimization and management of DDD paced patients with dilated cardiomyopathy Pacing Clin Electrophysiol. 1994;17(11 Pt 2):1984-1988.

3. Pearson AC, Janosik DL, Redd RR, et al. Doppler echocardiographic assessment of the effect of varying atrioventricular delay and pacemaker mode on left ventricular filling. Am Heart J. 1988;115(3):611-621.

4. Toda N, Ishikawa T, Nozawa N, et al. Doppler index and plasma level of atrial natriuretic hormone are improved by optimizing atrioventricular delay in atrioventricular block patients with implanted DDD pacemakers. Pacing Clin Electrophysiol. 2001;24(11):1660-1663.

5. Barold SS, Ilercil A, Herweg B. Echocardiographic optimization of the atrioventricular and interventricular intervals during cardiac resynchronization. Europace. 2008;10 Suppl 3:iii88-iii95.

6. Whinnett ZI, Davies JE, Willson K, et al. Haemodynamic effects of changes in atrioventricular and interventricular delay in cardiac resynchronisation therapy show a consistent pattern: analysis of shape, magnitude and relative importance of atrioventricular and interventricular delay. Heart. 2006;92(11):1628-1634.

7. Tei C, Nishimura RA, Seward JB, et al. Noninvasive Dopplerderived myocardial performance index: correlation with simultaneous measurements of cardiac catheterization measurements. J Am Soc Echocardiogr. 1997;10(2):169-178.

8. Gola A, Pozzoli M, Capomolla S, et al. Comparison of Doppler echocardiography with thermodilution for assessing cardiac output in advanced congestive heart failure. Am J Cardiol. 1996;78(6):708-712.
9. Ishikawa T, Sumita S, Kimura K, et al. Prediction of optimal atrioventricular delay in patients with implanted DDD pacemakers. Pacing Clin Electrophysiol. 1999;22(9):1365-1371.

10. Ritter P, Padeletti L, Gillio-Meina L, et al. Determination of the optimal atrioventricular delay in DDD pacing. Comparison between echo and peak endocardial acceleration measurements. Europace. 1999;1(2):126-130.

11. Meluzin J, Novak M, Mullerova J, et al. A fast and simple echocardiographic method of determination of the optimal atrioventricular delay in patients after biventricular stimulation. Pacing Clin Electrophysiol. 2004;27(1):58-64.

12. Jansen AH, Bracke FA, van Dantzig JM, et al. Correlation of echo-Doppler optimization of atrioventricular delay in cardiac resynchronization therapy with invasive hemodynamics in patients with heart failure secondary to ischemic or idiopathic dilated cardiomyopathy. Am J Cardiol. 2006;97(4):552-557.

13. Morales MA, Startari U, Panchetti L, et al. Atrioventricular delay optimization by doppler-derived left ventricular $\mathrm{dP} / \mathrm{dt}$ improves 6-month outcome of resynchronized patients. Pacing Clin Electrophysiol. 2006;29(6):564-568.

14. Gervais R, Leclercq C, Shankar A, et al. Surface electrocardiogram to predict outcome in candidates for cardiac resynchronization therapy: a sub-analysis of the CARE-HF trial. Eur J Heart Fail. 2009;11(7):699-705.

15. Aboulenein J, Tawfik M, Maaty A, et al. Optimizing The Atrioventricular Delay In Dual Chamber Pacemakers; Is It Worth? J Cardiol Curr Res. 2016;5(3).

16. Sawhney NS, Waggoner AD, Garhwal S, et al. Randomized prospective trial of atrioventricular delay programming for cardiac resynchronization therapy. Heart Rhythm. 2004;1(5):562-567.

17. Ellenbogen KA, Gold MR, Meyer TE, et al. Primary results from the smart delay determined $\mathrm{AV}$ optimization: a comparison to other $\mathrm{AV}$ delay methods used in cardiac resynchronization therapy (SMARTAV) trial: a randomized trial comparing empirical, echocardiographyguided, and algorithmic atrioventricular delay programming in cardiac resynchronization therapy. Circulation. 2010;122(25):2660-2668.

18. Ritter P, Dib JC, Lelievre T. Quick determination of the optimal AV delay at rest in patients paced in DDD mode for complete AV block. Eur JCPE. 1994;4:163.

19. Gorcsan J, Abraham T, Agler DA, et al. Echocardiography for cardiac resynchronization therapy: recommendations for performance and reporting-a report from the American Society of Echocardiography Dyssynchrony Writing Group endorsed by the Heart Rhythm Society. $J$ Am Soc Echocardiogr. 2008;21(3):191-213.

20. Antonini L, Auriti A, Pasceri V, et al. Optimization of the atrioventricular delay in sequential and biventricular pacing: physiological bases, critical review, and new purposes. Europace. 2012;14(7):929-938.

21. Brignole M, Auricchio A, Baron-Esquivias G, et al. 2013 ESC guidelines on cardiac pacing and cardiac resynchronization therapy: the task force on cardiac pacing and resynchronization therapy of the European Society of Cardiology (ESC). Developed in collaboration with the European Heart Rhythm Association (EHRA). Europace. 2013;15(8):1070-1118.

22. Connolly SJ, Kerr CR, Gent M, et al. Effects of physiologic pacing versus ventricular pacing on the risk of stroke and death due to cardiovascular causes. N Engl J Med. 2000;342(19):1385-1391.

23. Rossvoll O, Hatle LK. Pulmonary venous flow velocities recorded by transthoracic Doppler ultrasound: relation to left ventricular diastolic pressures. J Am Coll Cardiol. 1993;21(17):1687-1696.

24. Fukuhara K, Hiroyuki OH, Terumasa KT, et al. Feasibility of a novel atrioventricular delay optimization method using transmitral and pulmonary venous flow in patients with sequential ventricular pacing or cardiac resynchronization therapy. $J$ Echocardiogr. 2015;13(2):52-58. 
25. Izumi C, Yoshida $\mathrm{K}$, Akasaka $\mathrm{T}$, et al. Improved visualization of pulmonary venous flow Doppler signal by intravenous injection of sonicated albumin. Am J Cardiol. 1996;78(5):598-600.

26. Okura $\mathrm{H}$, Yoshida $\mathrm{K}$, Akasaka $\mathrm{T}$, et al. Improved transvalvular continuous-wave Doppler signal intensity after intravenous albunex injection in patients with prosthetic aortic valves. $J$ Am Soc Echocardiogr. 1997;10(6):608-612.
27. Okura H, Fuyuki H, Kubo T, et al. Noninvasive diagnosis of ischemic and nonischemic cardiomyopathy using coronary flow velocity measurements of the left anterior descending coronary artery by transthoracic Doppler echocardiography. J Am Soc Echocardiogr. 2006;19(5):552-558.

28. Okura H, Takada Y, Yamabe A, et al. Prevalence and correlates of physiological valvular regurgitation in healthy subjects. Circ J. 2011;75(11):2699-2704. 